paracetamol $650 \mathrm{mg}$ was more effective than dextropropoxyphene $65 \mathrm{mg}$ for the relief of pain following episiotomy. No greater benefit was derived by using the two drugs concomitantly, and dextropropoxyphene alone was no better than a placebo. The analgesic sffectiveness of Distalgesic is therefore largely due to its paracetamol content.

There is no doubt that Distalgesic is popular among patients and it is common knowledge among general practitioners that once Distalgesic has been prescribed for any length of time for whatever type of pain-but particularly in musculoskeletal disease, where psychosomatic factors are so prevalent-it becomes increasingly difficult to stop or change it. But this resistance by the patient is not due to the analgesic effectiveness of Distalgesic.

There are many reports in the literature ${ }^{4}{ }^{6}$ that prolonged use of dextropropoxyphene leads to dependence. Your own leading article $^{7}$ emphasises this and highlights the growing addiction to this drug, especially in the United States. It is this property of the drug and not its analgesic potency which makes it so popular.

Alan A Morgan

Ilford, Essex

Eddy, N B, et al, Bulletin of the World Health Organization, 1969, 40, 1 . National Academy of Sciences-National Research
Council Panel on Drugs for Relief of Pain. Medical

Letter on Drugs and Therapeutics, 1970, 12, 5 .
Hopkinson, $\mathrm{J} \mathrm{H}$, et al, fournal of Clinical Pharma

cology, 1973, 13, 251 .

eventeenth Report of the WHO Expert Committee on Drug Dependence. World Health Organization Technical Report Series, No 437, 1970.

Claghorn, J L, and Schooler, J C, fournal of the

American Medical Association, 1966, 196, 1089.

70,773 .
British Medical fournal, 1977, 1, 668.

\section{Human milk banking}

SIR,-Our recent studies in Oxford on human milk banking (13 January, p 80) have shown that if human milk is collected into sterile vessels and stored in the donor's domestic refrigerator for three to four days before being transported to the special care baby unit, then accurate pasteurisation eliminates potentially pathogenic organisms. These studies were conducted during the winter months of 1977 . Over the period May to August 1978 we found that the prepasteurisation bacterial counts of donated milk became progressively higher than we had observed before, perhaps relating to less effective domestic refrigeration at higher summer temperatures. It is well established that the initial bacterial count in milk determines the efficacy of pasteurisation, and the elevated prepasteurisation bacterial counts from July to mid-August 1978 resulted in nine out of 20 consecutive pasteuriser runs $(45 \%)$ failing to eliminate potentially pathogenic organisms Escherichia coli and Staphylococcus aureus (table). While these residual potential pathogens in the nine samples were present in low numbers after pasteurisation $\left(<2 \times 10^{2}\right.$ viable colony-forming units $/ \mathrm{ml}$ ), we considered that this milk was unsuitable for feeding to preterm neonates, though others have been prepared to use milk contaminated with $S$ aureus in these numbers. ${ }^{12}$

In view of our findings we elected to change our milk-banking procedure, and donors were instructed to store their milk after collection in the freezer compartment of their refrigerator, each 24-hour collection being stored in a separate sterile container. After the intro-
Post-pasteurisation bacteriology* of milk stored 3-4 days in a domestic refrigerator compared with milk stored up to 7 days in the frozen state: 20 pasteurisation runs in each group.

\begin{tabular}{lccc}
\hline & $\begin{array}{c}\text { No } \\
\text { growth }\end{array}$ & $\begin{array}{c}\text { Unlikely } \\
\text { onthogens } \\
\text { only }\end{array}$ & pathogens \\
\hline $\begin{array}{l}\text { Home } \\
\text { refrigeration } \\
\text { freezing }\end{array}$ & 5 & 6 & 9 \\
\hline
\end{tabular}
*Anaerobic and aerobic cultures performed on $0.2 \mathrm{ml}$
inocula into standard media: limit of detection, 5 viable colony-forming units ml of milk.

$* * S$ albus, Lactobacilli, Bacillus spp, $S$ faecalis.
${ }^{*} S$.

duction of home freezing (rather than refrigeration) there was an immediate marked reduction in the prepasteurisation bacterial flora of the milk, and following the next 20 consecutive pasteurisation runs during late August and September we were unable to isolate any potential pathogens. Indeed, all but one of the 20 cultures were "sterile" (fewer than five viable colony-forming units $/ \mathrm{ml}$ ) following pasteurisation. The difference between frozen stored and refrigerator stored milk, with respect to the efficacy of pasteurisation in eliminating potential pathogens, was highly significant $(\mathbf{P}<0.001$ by Fisher's exact probability test).

Human milk banking is still a poorly defined science and the effects of introducing each new procedure need to be assessed carefully. Previously we had tried to avoid prepasteurisation freezing of milk since Gibbs et al demonstrated that freezing may damage antimicrobial components in milk. ${ }^{3}$ However, Gibbs showed that these effects were related to the length of time the milk was stored in the frozen state, ${ }^{3}$ and we have therefore chosen not to allow home storage to exceed seven days. Further work is needed to evaluate the effects of freezing on human milk, but the studies reported here indicate that home freezing of donor milk in some circumstances is of value as an adjunct to precision pasteurisation.

University Department of

A LuCas Paediatrics

ALISON SMITH

J D BAUM

D G DAY

Pathology Department,

Pathology Department,
John Radcliffe Hospital,
Oxford

Williamson, S, et al, British Medical fournal, 1978, 1,

'2ucas, A, Goddard, P, Baum, J D, British Medical

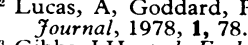

Gibbs, J H, et al, Early Human Development, 1977, 1,
227.

Norethisterone oenanthate as an

injectable contraceptive

SIR,-In a recent non-comparative clinica trial at King's College Hospital, London, Dr O F Giwa-Osagie and his colleagues (24 June, p 1660) reported on the use of norethisterone oenanthate (NET-OEN), $200 \mathrm{mg}$ given at eight-week intervals, in a sample of 295 women observed for 1606 months. The authors reported no pregnancies and very low termination rates for amenorrhoea.

The World Health Organisation's Special Programme of Research, Development and Research Training in Human Reproduction is currently conducting a multinational randomised comparative controlled clinical trial of depot-medroxyprogesterone acetate (DMPA) and NET-OEN in 11 countries. The injections of NET-OEN are given at eight-weekly intervals for the first six months of therapy, and then $50 \%$ of the NET-OEN subjects are randomly assigned to a 12-weekly injection schedule while the other $50 \%$ of subjects remain on the eight-weekly regimen. By 1 August last year, 1186 subjects had received NET-OEN and 13344 women-months of experience had been accumulated.

Since the WHO trial is an ongoing study, detailed findings cannot be released at this juncture. However, contrary to the results presented by Giwa-Osagie et al, there have been a number of pregnancies among women receiving NET-OEN at eight-week intervals. Furthermore, the termination rates for amenorrhoea among subjects receiving eightweekly NET-OEN injections is substantially higher than that reported by Giwa-Osagie et al, and higher than the rates observed in a previous. WHO trial, in which NET-OEN was administered at 12-week intervals.

The discrepancy between the findings of the ongoing large-scale WHO trial and the King's College Hospital study illustrate the problems that may arise with the interpretation of results derived from small samples, especially if the duration of observation is limited. From table III of the paper by Giwa-Osagie et al, it is clear that $70 \%$ of the total duration of observation on the 295 subjects entering the trial was attributable to women who had used the drug for six months or less.

It can be misleading to present cumulative data for prolonged periods of observation, such as one year, when most of the information is derived from a shorter duration of study. This is particularly a problem with long-acting injectable contraceptives, as amenorrhoea and the infrequent follow-up contacts may lead to delays in the diagnosis of pregnancies.

R H GRAY

Task Force on Long-Acting Systemic Agents for the Regulation of Fertility and WHO Special Programme of Research in Human Reproduction, World Health Organisation,

Geneva

World Health Organisation; Contraception, 1977, 15 513; and 1978, 17, 395.

\section{Mumps deafness}

SIR,-The paper by Mr G A Westmore and colleagues (6 January, p 14) will be interpreted as showing that deafness in mumps is due to the presence of the virus in the perilymph, entering the inner ear by haematogenous spread. It is surely quite impossible, however, for a generalised viraemia to cause the unilateral or sudden deafnesses that occur in viral infections.

In mumps, virus is very hard to demonstrate in the blood as opposed to other body fluids. ${ }^{1}$ It is far more likely that the widespread viral dissemination occurs via the lymphatic vessels, and that this is how it can get into the cerebrospinal fluid in cases of meningoencephalitis. Since there is cervical lymphoedema in mumps, there will be an ascending stasis and large hydrostatic pressures may be transmitted backwards into the labyrinthine fluids, since one of the CSF drainage routes into the cervical lymph nodes passes through the inner ear. ${ }^{2}$ This will predispose to a sudden rupture of a labyrinthine window. A stapedectomised ear is likely to have a weak oval 
window seal. The fact that the authors did not find a perilymph fistula does not exclude this possibility as they can heal very rapidly, as after routine stapedectomy. I note that tinnitus and nausea had resolved by the time of surgery. Incidentally, experimental inoculation of mumps virus at the oval window does not lead to labyrinthitis in monkeys. ${ }^{3}$

A G GoRDON

London SE5

' Overman, J R, Archives of Internal Medicine, 1958, $102,354$.

Foldi, M, American Heart Yournal, 1977, 93, 121.

Karmody, C S, Annals of Otology, Rhinology and

\section{Abdominal and thoracic pressures during defecation}

SIR,-Following the article by Dr S S Fedail and others (13 January, $p$ 91) entitled "Abdominal and thoracic pressures during defecation" I would like to comment on the method and interpretation of the results.

Firstly, the technique of locating the diaphragm by the point of respiratory reversal on the manometry tracing is somewhat erroneous. The point of respiratory reversal is normally at the upper end of the highpressure zone (sphincter + diaphragm) above the lower oesophageal sphincter component. The diaphragmatic component of the highpressure zone normally appears at the lower (distal) aspect. However, the proximal tube would undoubtedly have been in the oesophagus and the pressures they measured would have accurately reflected the difference between stomach and thorax. There is naturally a significant difference between these results, but the important factor is the gastrooesophageal pressure difference. There was no significant difference between the squatters and the sitters. The authors fail to mention the important closing and antireflux mechanisms that occur at the gastro-oesophageal junction -for example, angle of implantation, mucosal flap, and right crura, all of which depend to a large extent on a positive intra-abdominal pressure.

In conclusion, I think that their idea is correct and our mode of defecation may well be a causative factor in the development of hiatal hernia. However, it is unwise to base the argument on such flimsy "not significant" evidence.

Thoracic Surgical Unit,

Papworth Hospital,

Cambridge

SIR,-Dr S S Fedail and others (13 January, p 91) put forward evidence to support the opinions of Muller ${ }^{1}$ and Burkitt and James ${ }^{2}$ that the pressure gradient between abdomen and thorax during straining at stool is an aetiological factor in hiatus hernia.

Although attractively simple, this does not withstand any detailed examination, since it fails entirely to explain why the oesophagus withdraws pari passu as the stomach advances up the mediastinum, as is the case in sliding hiatus hernia, where the oesophagogastric junction is always at the apex of the herniated stomach. The sliding type of hernia comprises the vast majority of all hiatus hernia. It fails also to explain the phenomenon of incompetence of the oesophagogastric sphincter, usually found in association with sliding hernia, but often found in its absence.

Damage to the hiatus-for example, surgical trauma-results not in the sliding type of hernia but in the paraoesophageal type, in which the fundus of the stomach, or a part of it, together with the oesophagogastric junction, are herniated into the lower mediastinum, without disturbance of function of the sphincter, and without shortening of the oesophagus. One would expect this type of hernia to result from a raised pressure gradient across the diaphragm, as in straining at stool; but it does not.

The prevalence of hiatus hernia in the West and its rarity in Africa invite the conclusion that a principal aetiological factor is diet. With this I agree; but I feel that this factor induces a disorder of oesophagogastric motility, the important feature being overactivity of the longitudinal muscle of the oesophagus. This leads to oesophageal shortening, which draws the stomach into the chest, producing the characteristic sliding hernia. It is this shortening-and thickening-which distracts, elongates, and renders incompetent the oesophagogastric sphincter. It is this shortening which often renders repair of the hernia difficult, and sometimes impossible. Surgical procedures which overcome the shortening or the tension in the oesophagus relieve the symptoms, although they need not necessarily reduce the hernia.

Unfortunately we have no means of measuring the activity of the longitudinal muscle of the oesophagus, despite having had elegant methods of measurement of the circular muscle for many years. As we cannot measure longitudinal muscle activity, we prefer to ignore it. But the longitudinal muscle is by far the most prominent feature of the oesophagus.

In short, sliding hiatus hernia is not pushed up into the chest by abdominal pressure; it is pulled up by the oesophagus. I have previously elaborated this, basing my views on surgical experience. $^{3}$

KenNeth S Mullard

Bletchington, Oxon

'Muller, C J B, South African Medical fournal, 1948, 22, 376.

Burkitt, D P, and James, P A, Lancet, 1973, 2, 128 Mullard, K S, Annals of the Royal College of Surgeon
of England, 1972, 50, 73 .

\section{Physical therapy in chronic bronchitis}

SIR,-Dr Margaret E Hodson and others (6 January, p 55) correctly observe that we did not separate the effect of intermittent positive pressure breathing (IPPB) and physiotherapy (2 December, $p$ 1525). The similar response of our treated and control groups suggests that this was an economic and wise decision, unles one considers these forms of treatment mutually antagonistic.

I would be surprised if any alteration in physiotherapy technique or method of administration of bronchodilator would produce better results than we obtained in either of our treatment groups, but shall await the evidence.

Your correspondents quoted an excellent paper by Shenfield et al as evidence that a bronchodilator is more efficient by IPPB than by aerosol. ${ }^{1}$ The actual title of the paper wa "The fate of nebulised salbutamol (Albuterol) administered by intermittent positive respiration to asthmatic patients." As the title suggests, it was concerned with the pharmacodynamics of inhaled salbutamol, and blood levels of the drug over eight hours and urine and faecal samples over three days. One could not suggest from the data presented in the paper that the use of IPPB with nebulised salbutamol was more effective than a pressurised aerosol alone, as the authors did in the summary. Furthermore, this paper referred to five convalescing asthmatics. We considered the paper by Berend et al more appropriate, as it concerned the disease we were studying and for that reason quoted it. ${ }^{2}$

The final point in the letter about the importance of physiotherapy and the use of a bronchodilator for those who have large volumes of secretions, is discussed in the Lancet editorial to which it refers. ${ }^{3}$

Service de Pneumologie,

DunCaN NewtoN Hôpital
Paris

${ }^{1}$ Shenfield, G M, et al, American Review of Respiratory Diseases, 1973, 108, 501

Berend, N, et al, British fournal of Diseases of the Chest, 1978, 72, 122.

${ }^{3}$ Newton, D A G, Lancet, 1979, 1, 49.

\section{An unsavoury custom?}

SIR,-It is one of the marks of a first-class hospital that its doctors and laboratory staff do not come to meals wearing their white coats. I know of no spread of an infection which was traced to this unsavoury custom; but why wait till something untoward occurs before discontinuing the practice where it still persists? Admittedly there are often administrative difficulties, notably in teaching hospitals where changing facilities would have to be found for numerous clinical students. The DHSS definitely frowns on this custom. If medical men do not feel concerned, perhaps the administrators in charge of hospital dining rooms and canteens ought to act and exclude clothing which might be contaminated from areas where food is served and consumed.

H LEHMANN

Department of Biochemistry, University of Cambridge

\section{Hazards of sulphinpyrazone}

SIR,-With reference to the letter on hazards of sulphinpyrazone (23-30 December, p 1786) I would like to make some comments.

Sulphinpyrazone being a pyrazole compound is related to phenylbutazone, which has been observed to potentiate the hypoglycaemic effect of the oral sulphonylureas. Hence sulphinpyrazone should be used with caution in patients on concomitant sulpha drugs, the sulphonylurea hypoglycaemic agents, and insulin.

Sulphinpyrazone is potent uricosuric agent, and alkalinisation of urine and high fluid intake are necessary at first to avoid crystalluria. In patients on phenylbutazone oedema occurs, due to a renal effect in reducing sodium and water excretion. Since sulphinpyrazone is closely related (a pyrazole) to phenylbutazone the salt retention as a side effect should also be borne in mind, for it may precipitate heart failure.

I endorse the note of caution of $\mathrm{Dr} D$ Mattingly and his colleagues that sulphinpyrazone is contraindicated in patients on oral anticoagulants. This drug is also contraindicated in patients with history of hypersensitivity 